\title{
Next-generation sequencing (NGS) as a diagnostic tool for retinal degeneration reveals a much higher detection rate in early-onset disease
}

\author{
Morag E Shanks, Susan M Downes, Richard R Copley, Stefano Lise, John Broxholme, Karl AZ Hudspith, \\ Alexandra Kwasniewska, Wayne IL Davies, Mark W Hankins, Emily R Packham, Penny Clouston, \\ Anneke Seller, Andrew OM Wilkie, Jenny C Taylor, Jiannis Ragoussis and Andrea H Németh
}

European Journal of Human Genetics (2013) 21, 1031; doi:10.1038/ejhg.2013.91

Correction to: European Journal of Human Genetics (2013) 21, 274-280; doi:10.1038/ejhg.2012.172

The authors would like to apologise for the following errors in Table 2.

Three references cited in Table 2 were absent from the final reference list:

ABCA4 N965S

Sun H, Smallwood PM, Nathans J: Biochemical defects in ABCR protein variants associated with human retinopathies. Nat Genet 2000; 26: $242-246$

CRX Y258X

Chau KY, Chen S, Zack DJ, Ono SJ: Functional domains of the conerod homeobox (CRX) transcription factor. J Biol Chem 2000; 275: 37264-37270.
GUCY2D R838H

Wilkie SE, Newbold RJ, Deery E et al: Functional characterization of missense mutations at codon 838 in retinal guanylate cyclase correlates with disease severity in patients with autosomal dominant cone-rod dystrophy. Hum Mol Genet 2000; 9: 3065-3073.

The dbSNP entry rs61749449 refers to N965D rather than N965S.

CRB1T, 745K should read CRB1, T745K.

Also, the following errors were found in Supplementary Table 2:

ABCA4 c.322C $>$ T should read c.3322C $>\mathrm{T}$

ABCA4 c.5882G $>$ C should read c.5882G $>$ A

ABCA4 c.6379C $>$ T should read c.6391G $>$ A

PRPF8 c.6926T $>$ G should read c.6926A $>$ C

\section{Mortality in neurofibromatosis 1: in North West England: an assessment of actuarial survival in a region of the UK since 1989}

D Gareth R Evans, Catherine O’Hara, Anna Wilding, Sarah L Ingham, Elizabeth Howard, John Dawson, Anthony Moran, Vilka Scott-Kitching, Felicity Holt and Susan M Huson

European Journal of Human Genetics (2013) 21, 1031; doi:10.1038/ejhg.2013.121

Correction to: European Journal of Human Genetics (2011) 19, In the first row of Table 4 the PMR (95\% CI) should be 4.1 (2.4, 6.4) 1187-1191; doi:10.1038/ejhg.2011.113 rather than $4.1(2.4,2.6)$. Also, the first sentence on page 1189 should read:

The authors would like to apologize for the following error that has been brought to their attention.

'...cardiovascular disease was reported four times more frequently in $\mathrm{NF} 1$ males (PMR $=4.1 ; 95 \% \mathrm{CI}, 2.4-6.4$ ) than the general population.' 